\title{
Diagnosis and treatment of refractory pituitary adenomas: a narrative review
}

\author{
Xiaohai Liu ${ }^{1,2 \#}$, Congxin Dai ${ }^{2,3 \#}$, Ming Feng ${ }^{2,4}$, Mingchu Li $^{1,2}$, Ge Chen ${ }^{1,2}$, Renzhi Wang ${ }^{2,4}$ \\ ${ }^{1}$ Department of Neurosurgery, Xuanwu Hospital Capital Medical University, Beijing, China; ${ }^{2}$ Chinese Pituitary Specialists Congress, Beijing, China; \\ ${ }^{3}$ Department of Neurosurgery, Tongren Hospital Capital Medical University, Beijing, China; ${ }^{4}$ Department of Neurosurgery, Peking Union Medical \\ College Hospital, Chinese Academy of Medical Sciences and Peking Union Medical College, Beijing, China \\ Contributions: (I) Conception and design: X Liu, R Wang; (II) Administrative support: G Chen, R Wang; (III) Provision of study materials or patients: \\ All authors; (IV) Collection and assembly of data: All authors; (V) Data analysis and interpretation: All authors; (VI) Manuscript writing: All authors; \\ (VII) Final approval of manuscript: All authors. \\ \#These authors contributed equally to this work. \\ Correspondence to: Renzhi Wang. Department of Neurosurgery, Peking Union Medical College Hospital, Chinese Academy of Medical Sciences and \\ Peking Union Medical College, Beijing 100730, China. Email: wangrz@126.com.
}

\begin{abstract}
Although aggressive pituitary adenomas (PAs) have been proposed and widely discussed for more than a decade, there is no general agreement regarding their definition, diagnosis or management. As one of the largest pituitary centers in China, we have diagnosed and treated more than fifty cases of aggressive PA and 3 pituitary carcinomas in the past 5 years and proposed a new term, i.e., refractory PAs, to define these adenomas. The definitions of aggressive and refractory PAs overlap with each other, though there are some differences between them. We interpret the definition for refractory PA in this review, emphasizing that more attention and early identification of these adenomas are needed. Although temozolomide (TMZ) has been used to treat pituitary carcinomas and refractory PA since 2006, which has significantly improved the prognosis of these patients, treatment of refractory PA is a tremendous challenge for endocrinologists and neurosurgeons. Overall, refractory PA is defined as PA with a Ki-67 labeling index $\geq 3 \%$, a faster growth rate than that of normal PA, infiltration of surrounding tissues, recurrence or regrowth in the early postoperative period, and continued growth and/or secretion of excessive hormones despite attempts to control it. These criteria for refractory PA are stricter than for aggressive PA. The diagnosis and treatment of refractory PA requires the collaboration of a multidisciplinary team to achieve the best results.
\end{abstract}

Keywords: Refractory pituitary adenomas; aggressive pituitary adenomas; diagnosis; Ki-67 index; temozolomide

Submitted Dec 06, 2020. Accepted for publication Mar 05, 2021.

doi: $10.21037 /$ gs-20-873

View this article at: http://dx.doi.org/10.21037/gs-20-873

\section{Introduction}

Pituitary adenomas (PAs) arising from adenohypophyseal cells account for approximately $15 \%$ of intracranial tumors (1). In the largest meta-analysis of autopsy studies comprising 18,902 examined pituitaries from 32 series (2), the mean prevalence of pituitary incidentaloma was $10.7 \%$ (range $1.5-31 \%$ ). However, the prevalence of clinically significant $\mathrm{PA}$ in the general population has recently been found to be 1/1,000 (3), indicating that most PAs maintain the characteristics of benign tumors and do not grow in size or secrete hormones over many years. Although most PAs can be cured or controlled surgically, pharmacologically and/or radiologically, a small set of PAs with a high Ki-67, rapid growth, infiltration of surrounding tissues, and early and multiple recurrences are refractory to conventional treatments such as surgery, drugs and radiotherapy. These PAs are notoriously difficult to manage and are referred to as aggressive PAs $(4,5)$. According to the European Society for Endocrinology (ESE) guidelines, the diagnosis of aggressive PA should be considered in patients with a 
radiologically invasive tumor and an unusually rapid tumor growth rate or clinically relevant tumor growth despite optimal standard therapies, including surgery, radiotherapy and conventional medical treatments (6). Aggressive PA can seriously affect the quality of life of the patient, and the prognosis of these patients is very poor. Consequently, early identification and acute diagnosis along with personalized treatment strategies for aggressive PA are crucial for appropriate management.

Although aggressive PAs have been proposed and widely discussed for more than a decade, there is no general agreement regarding their definition, diagnosis or management. As one of the largest pituitary centers in China, we have diagnosed and treated more than fifty cases of aggressive PA and 3 pituitary carcinomas in the past 5 years, and we proposed the new term "refractory PA" to define these adenomas $(7,8)$. Although the definitions of aggressive and refractory PAs overlap, the definition of refractory PA includes radiological findings and histopathological and clinical features, in particular, emphasizing the importance of the $\mathrm{Ki}-67$ index and growth velocity, which are stricter criteria than for aggressive PA, with a worse prognosis. We present the following article in accordance with the Narrative Review reporting checklist (available at http://dx.doi.org/10.21037/gs-20-873).

\section{Definition of refractory PA}

Although only a very minority of PA patients develop aggressive adenomas, many efforts in the past two decades have been made to improve the definition and diagnostic process of these Pas that show aggressive behavior and poor prognosis. The World Health Organization (WHO) classification of PAs in 2004 categorized these tumors as typical, atypical, or carcinoma, and atypical adenoma was characterized as follows: a Ki-67 labeling index $\geq 3 \%$, increased mitotic count, or elevated $\mathrm{p} 53$ and immune response activities (9). Atypical PAs comprise 2.7-15\% of the total number of all PAs (10). However, in many subsequent studies, the diagnosis of atypical PA did not indicate the biological behavior of the tumor or the poor prognosis of the disease $(11,12)$. Therefore, the definition of atypical PA was removed, and the concept of aggressive PA was formally put forward in the 4th WHO classification of PA in 2017, in which the assessment of proliferative activity and invasion was recommended for diagnosing aggressive PA (13). High-risk PAs, including sparsely granulated somatotroph adenoma, lactortroph adenoma in males, silent Crooke cell corticotroph adenoma, and Pit-1positive plurihormonal adenoma, previously termed silent subtype 3, have the common characteristics of rapid growth, radiological invasion, and a high $\mathrm{Ki}-67$ proliferation index; they also tend to be recurrent and resistant to conventional therapy (13).

Regardless of whether PAs are classified as typical or atypical and aggressive or nonaggressive, such categorization seeks to identify the malignant behavior of PA without cerebrospinal and/or systemic metastasis, which ultimately results in a poor prognosis. Although aggressive PAs are considered in patients with a radiologically invasive tumor and an unusually rapid growth rate or clinically relevant tumor growth despite optimal standard therapies, as described above, the definition of aggressiveness for pituitary tumors is rather confusing, particularly because of the lack of specific histopathological features (14). Furthermore, aggressiveness and invasiveness are interpreted differently by individual clinicians, and these terms are often used interchangeably (15). Nevertheless, most nonfunctioning PAs extending into the cavernous sinus are neither aggressive nor invasive, and cavernous sinus extension of PAs may be caused by weakness of the medial wall of the cavernous sinus (16). In our case series, total resection through extended transsphenoidal surgery was achieved for a substantial number of invasive Pas, with no recurrence after a long followup time (Figure 1) (17). Overall, a more accurate definition and objective diagnostic criteria are needed.

In 2016, we diagnosed and treated more than fifty cases of aggressive PAs and proposed a new term, "refractory PA", to define these adenomas (7). By considering research progress and increasing numbers of patients with refractory Pas being treated, we summarized the diagnostic criteria for refractory PA as follows: (I) the tumor infiltrates adjacent structures according to radiological results or intraoperative findings; (II) the Ki-67 index is greater than $3 \%$, and the tumor growth velocity in volume is $\geq 2 \%$ per month; (III) conventional treatments fail to control tumor growth and/ or hormonal hypersecretion; (IV) tumor recurrence or regrowth occurs within 6 months after surgery; (V) tumor growth and/or hormone hypersecretion seriously affect the patient's quality of life and can even be life-threatening; and (VI) no intracranial or intraspinal metastasis or distant metastasis occurs $(7,8)$. Although the term refractory is not yet accepted by most pituitary specialists, the definition of refractory PA includes radiological findings and histopathological and clinical features, in particular, emphasizing the importance of the Ki-67 index and growth velocity, which are stricter criteria than for aggressive PA, 

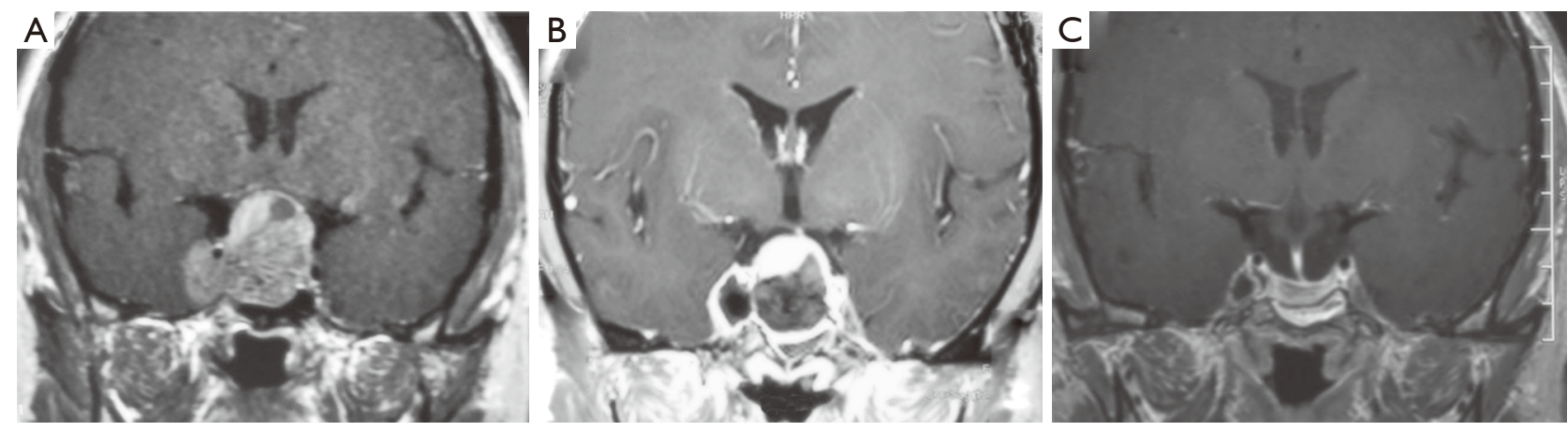

Figure 1 Total resection through extended transsphenoidal surgery was achieved for a 26-year patient with cavernous sinus invasive pituitary adenoma. (A,B) (4 days after surgery), with showed no recurrence after 18 months $(C)$.

with a worse prognosis.

$\mathrm{Ki}-67$ is an immunohistochemical marker used for prognostic prediction in PA (18). The Ki-67 index of aggressive PAs is much higher than that of benign PAs (19), indicating its importance compared with other histological and clinical markers, such as widely positive $\mathrm{p} 53$ expression and mitotic count $\geq 2 / 10$ high-power fields. In another retrospective study by Trouillas et al. (20), Ki-67 and other proliferation parameters were added to define aggressive tumors. PAs were classified into 5 grades (grade 1a: noninvasive, $1 \mathrm{~b}$ : noninvasive and proliferative, $2 \mathrm{a}$ : invasive, $2 \mathrm{~b}$ : invasive and proliferative, and 3: metastatic) based on tumor size, type, invasion, p53 and cell cycle markers (Ki67). After 8 years of follow-up, invasive and proliferative tumors (grade $2 \mathrm{~b}$ ) had a relatively poor prognosis compared to noninvasive tumors (20). Moreover, Petry et al. (21) showed that the Ki-67 index was predictive of regrowth in nonfunctioning adenomas, and in a recent study, patients with the highest Ki67 index (30\%) had a risk of developing pituitary carcinoma, indicating that more attention should be paid to a Ki67 index $\geq 3 \%$. Nonetheless, it remains unclear whether a specific Ki-67 index can be used as a diagnostic marker for refractory PA, and more research is needed. In our previous study, we proposed using not only the cutoff value of $\geq 3 \%$ for Ki- 67 but also the tumor growth velocity $\geq 2 \%$ per month, as rapid growth rate is an important sign of refractory PA (7). Additionally, tumor recurrence or regrowth can occur within a short period (6 months) after surgical resection, even when complete resection is achieved (7). Therefore, rapid deterioration of the clinical manifestations and a sudden increase in hormone levels are also indications of the progression of functional pituitary tumors.

To date, there are no specific molecular biomarkers to indicate refractory PA. Indeed, attempts to establish predictive and/or prognostic biomarkers of clinical aggressiveness for refractory PA remain largely unsuccessful due to a lack of understanding of their pathogenesis and a lack of case series. However, the histopathological structure of refractory PA cells is not significantly different from that of benign PA cells or pituitary carcinoma cells (22). Morphologic features associated with refractoriness, including nuclear and cellular pleomorphism or increased mitotic activity, are commonly present but are not necessarily diagnostic for refractory PA; a high Ki-67 labeling index $\geq 3 \%$ and dural and/or bony invasion are necessary but not adequate for the diagnosis of refractory $\mathrm{PA}$, as discussed above.

Both aggressive and refractory tumors involve clinically relevant tumor growth, despite optimal standard therapies. Nevertheless, the definitions and diagnostic criteria for aggressive disease do not provide an objective criterion for rapid growth, which easily leads to different judgments among clinicians. The definition of refractory includes a tumor growth velocity $\geq 2 \%$ per month, which is used to determine rapid growth. Although tumor growth velocity $\geq 2 \%$ per month still it does provide an objective criterion for rapid growth. The differences and similarities among atypical, aggressive, high-risk and refractory PA are shown in Table 1.

Although characteristics of refractory RA overlap those of aggressive and resistant adenomas, there are some differences. As an intermediate stage in phenotype between benign PA and pituitary carcinoma, the definition of refractory PA is based not only on the clinical and pathological features of the adenoma, the response to conventional therapy and the perspective of the patient's clinical outcome but also on the importance of rapid growth patterns, even under intensive treatment. In 
Table 1 The different characteristics of atypical, aggressive, high-risk and refractory PA

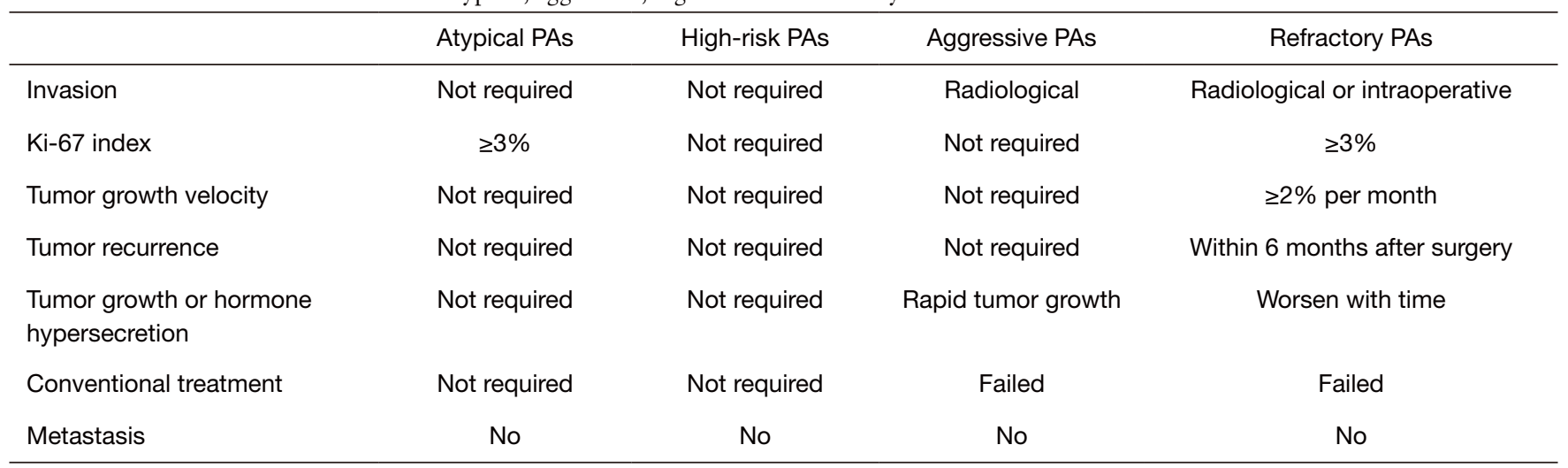

contrast, aggressive PAs only exhibit clinical features and poor prognosis. By reviewing 102 patients with aggressive Ceccato et al. (23) also proposed a new and comprehensive definition of aggressiveness based upon radiological, clinical and pathological features, similar to our proposal.

Therefore, compared with "aggressive" PA, refractory PA might more accurately represent the clinical features of the malignant behavior of PA without cerebrospinal metastasis, including radiological findings, pathological features and dynamic clinical behavior. Regardless, stricter and more accurate clinical characteristics of refractory PA are needed for diagnosis.

\section{Molecular tumorigenesis of the aggressive behavior of refractory PA}

Genetic or epigenetic abnormalities, disorders in paracrine growth factor signaling, and changes in the pituitary microenvironment can result in the activation of pituitaryspecific oncogenes or inactivation of tumor suppressor genes, leading to sustained cell proliferation (24). Although mutations in GNAS [the gene encoding the stimulatory alpha subunit of the guanine nucleotide-binding protein (G-protein) that stimulates adenylate cyclase] and USP8 (ubiquitin carboxyl terminal hydrolase 8) occur in subgroups of nonfamilial growth hormone-secreting tumors and corticotropin-secreting tumors, respectively (25), the underlying cause at the molecular level of other PAs remains unclear, and the genetic evaluation of PAs is rarely helpful for management. Moreover, the process of malignant transformation and progression from benign PA to refractory $\mathrm{PA}$ remains unclear, and the progression from aggressive/ malignant characteristics to pituitary carcinoma is poorly understood (26). Although the incidence of malignant progression of PA is very rare, once it occurs, the tumor becomes aggressive, namely, refractory PA or pituitary carcinoma with either craniospinal dissemination or systemic metastases.

For young patients with a clear family history of pituitary and endocrine tumors, germline genetic testing is recommended to search for evidence of genetic changes. PAs associated with multiple endocrine neoplastic syndrome type 1 (MEN1) and aromatic receptor-interacting protein (AIP) gene mutations tend to be more refractory $(27,28)$. Other genes associated with susceptibility to PAs include GPR101 (x-linked acromegaly gigantism, XLAG), cyclin kinase inhibitor p27Kip1 (multiple endocrine neoplasia type 4, multiple endocrine neoplasia type 4, MEN4), PRKAR1A (Carney complex), GNAS (McCune-Albright syndrome), neurofibromatosis type 1, SDHx mutation and DICER1 syndrome $(28,29)$.

\section{Treatment}

\section{Importance of multidisciplinary collaboration for diagnosis and treatment}

The diagnosis and treatment of refractory PA requires the collaboration of a multidisciplinary team (MDT) to achieve the best results (30). Therefore, once a diagnosis of refractory $\mathrm{PA}$ is suspected, the diagnosis and treatment must rely on the cooperation of members in the MDT, which should consist of doctors with expertise in neurosurgery, endocrinology, radiotherapy, radiology, pathology, oncology, neuroophthalmology and other specialties. The tasks of the MDT include not only patient diagnosis and comprehensive endocrine assessment but also the 
selection of optimal treatment modalities, determination of appropriate combinations of different treatments and lifelong follow-up.

\section{Surgical treatment}

As refractory PAs continue to grow even after multiple and combined operations, conventional drug therapy and radiotherapy, MDTs need to discuss the possibility of surgical debulking (4). In fact, surgical debulking can achieve subtotal resection, which alleviates the pressure of the tumor on the optic nerve, optic chiasma, hypothalamus, brain stem and other important structures and reduces reverse hydrocephalus, and it may also enhance the effect of adjuvant therapy, such as radiotherapy or TMZ.

When considering surgical debulking, the patient's systemic status and the possibility of surgical complications should be comprehensively evaluated, and the risks and benefits of surgery should be weighed wisely. As studies have suggested that surgeons with extensive experience in pituitary surgery have a lower incidence of surgical complications and mortality, it is recommended that neurosurgeons with extensive surgical experience in PA perform the operation (31). An extended endoscopic approach in which wide exposure and direct visualization can be achieved can facilitate the removal of tumors that invade the cavernous sinus and other sites, but it should be noted that such an approach might result in significant arterial damage, cranial nerve damage and postoperative cerebrospinal fluid leakage (32). Assistive techniques such as intraoperative navigation, intraoperative MRI, vascular Doppler ultrasound, neuroelectrophysiological monitoring and cerebrospinal fluid leakage repair can improve the resection rate and safety of transsphenoidal surgery (33).

\section{Radiotberapy}

All patients with refractory PA with a residual tumor should consider the possibility of radiation therapy. However, as most patients have previously received radiation therapy once or even more than twice, the patient's radiotherapy tolerance should be comprehensively assessed before considering radiotherapy. Once it has been determined that a patient with refractory PA can tolerate radiation therapy, the therapy alone or combined with TMZ should be utilized. Despite very limited data regarding radiotherapy approaches for refractory PA, both conventional external beam radiotherapy and stereotactic radiosurgery are effective at controlling or pituitary carcinoma growth (34). Conventional external beam radiotherapy has been used for several decades and has shown good clinical efficacy at a total dose of 45-54 Gy, which is divided into 25-30 exposures (35). However, stereotactic radiosurgery is becoming more popular for patients with irregular tumor shapes, especially those invading the visual pathway, pituitary stalk, brain stem and other important structures (36). Different modes of stereotactic irradiation are available, including linear accelerators (such as LINAC and CyberKnife), multisource cobalt-60 devices (such as gamma knives), and proton beam accelerators $(37,38)$. When the tumor is adjacent to the visual pathway, stereotactic radiosurgery can be divided into $3-5$ sessions, which not only protects the adjacent structures at risk of injury but also increases the radiation dose delivered to the tumor to control its growth (39). Stereotactic radiosurgery generally requires the tumor edge to be at least $3-5 \mathrm{~mm}$ away from the visual pathway, and the maximum diameter of the tumor should not exceed $3 \mathrm{~cm}$ (40). Although there have been no randomized controlled trials (RCTs) comparing these approaches, it has been reported that stereotactic radiosurgery has a certain effect on residual RPA with strong proliferative activity after conventional radiotherapy (41).

In general, the risks associated with radiotherapy, including hypopituitarism, optic neuropathy and other cranial neuropathies, should be considered. Nearly every patient treated with radiation can develop one or more pituitary hormone deficiencies given a long period of follow-up (42). Hypopituitarism itself is a risk factor for premature death. Thus, it is necessary to provide education and guidance to patients regarding the need to undergo regular and comprehensive endocrine examinations to assess pituitary function and receive hormone replacement therapy in a timely manner. Due to the lack of support from prospective studies regarding the optimal radiotherapy dose for refractory PA, the dose currently used may not effectively achieve the goal of long-term tumor growth control, and further studies are needed (43).

\section{TMZ treatment}

Temozolomide (TMZ) is an alkylating agent that can readily cross the blood-brain barrier and inhibit the growth of tumor cells in each stage of the cell cycle; it is suitable for slow-growing PAs (44). Successful treatment of PA with TMZ was first reported in $2006(45,46)$. To date, more than 300 cases of PA treated with TMZ have been reported, including a cohort of 166 patients in the 
international survey performed by the ESE, in which the response rate was approximately $60 \%(47,48)$. Thus, TMZ is recommended as a first-line treatment for refractory PA and pituitary carcinoma according to the European Society of Endocrinology Clinical Practice Guidelines. TMZ treatment should be considered once the diagnosis of refractory $\mathrm{PA}$ is made.

TMZ acts via methylation of the $\mathrm{O} 6$ position of guanine in DNA to form a potent cytotoxic DNA adduct, which causes a disturbance in the DNA sequence, leading to DNA double-strand breakage (49). The endogenous DNA repair protein $\mathrm{O}(6)$-methylguanine methyltransferase (MGMT) removes the methyl group, potentially counteracting the cytotoxic effects of TMZ (50). Low MGMT expression due to methylation of the MGMT promoter is related to the strong response of glioblastoma to TMZ treatment (51). In the ESE survey with 166 PA cases, those with low MGMT expression often achieved regression, whereas tumors with high MGMT expression more often showed no response (48). Therefore, immunohistochemical staining for MGMT should be carried out on tissue samples of refractory PA, with high staining positivity indicating a poor prognosis. As MMR is involved in the removal of DNA base mismatches caused either by DNA replication errors or DNA damage, cells deficient in MMR are significantly less sensitive to TMZ than cells with intact MMR (52). Loss of MSH2 and MSH 6 was observed in a single patient who had a rapid development of resistance to TMZ (53), and more studies are needed to examine the involvement of MMR proteins in refractory PA treated with TMZ.

\section{Other treatments}

Other agents should be considered for refractory PA that fails to respond to TMZ or acquired TMZ resistance after an effective response. At present, the most commonly used drug combinations for the treatment of RPA and $\mathrm{PC}$ are lomustine (CCNU) and 5-fluorouracil (5-FU), but this regimen is only effective at controlling tumor growth for a short period of time (54). Targeted therapy studies have found that the RAF/MEK/ERK, PI3K/Akt/ mTOR and EGFR pathways, which are associated with tumor occurrence and progression, are upregulated in PA $(55,56)$. However, in one of these studies, control of tumor progression failed in all eight cases of refractory PA or pituitary carcinoma treated with everolimus (55). The tyrosine kinase inhibitor lapatinib was found to control tumor progression in two refractory pituitary prolactin adenomas (57); additionally, targeting vascular endothelial growth factor (VEGF) using bevacizumab has been attempted and shown to be successful in some patients (58). Programmed death ligand 1 (PD-L1) is expressed in refractory $\mathrm{PA}$, and treatment with the immune checkpoint inhibitors ipilimumab and nivolumab has been reported for ACTH-secreting PAs with significant tumor shrinkage and hormonal response (59). A clinical study of peptide receptor radionuclide therapy (PPRT) for pituitary neuroendocrine neoplasms has yet to be carried out.

\section{Conclusions}

Compared with aggressive PA, the definition and diagnostic criteria for refractory PA are stricter. Once the diagnosis of refractory PA is suspected, the patient should be referred to regional pituitary centers with an experienced MDT and undergo aggressive treatment and lifelong follow-up. Additionally, investigations into the mechanisms underlying the tumorigenesis and progression of PA are urgently needed for additional treatments.

\section{Acknowledgments}

Funding: Financial support for this study was provided by the Scientific Research Project of Capital Health Development in 2018 (grant number: 2018-4-4018). The funding institutions had no role in the design of the study, data collection and analysis, decision to publish, or preparation of the manuscript.

\section{Footnote}

Reporting Checklist: The authors have completed the Narrative Review reporting checklist. Available at http://dx.doi.org/10.21037/gs-20-873

Conflicts of Interest: All authors have completed the ICMJE uniform disclosure form (available at http://dx.doi. org/10.21037/gs-20-873). The authors have no conflicts of interest to declare.

Ethical Statement: The authors are accountable for all aspects of the work in ensuring that questions related to the accuracy or integrity of any part of the work are appropriately investigated and resolved. Written informed consent was obtained from all patients according to institutional guidelines for publication of this review and 
any accompanying images.

Open Access Statement: This is an Open Access article distributed in accordance with the Creative Commons Attribution-NonCommercial-NoDerivs 4.0 International License (CC BY-NC-ND 4.0), which permits the noncommercial replication and distribution of the article with the strict proviso that no changes or edits are made and the original work is properly cited (including links to both the formal publication through the relevant DOI and the license). See: https://creativecommons.org/licenses/by-nc-nd/4.0/.

\section{References}

1. Gittleman H, Ostrom QT, Farah PD, et al. Descriptive epidemiology of pituitary tumors in the United States, 2004-2009. J Neurosurg 2014;121:527-35.

2. Molitch ME. Pituitary incidentalomas. Best Pract Res Clin Endocrinol Metab 2009;23:667-75.

3. Daly AF, Rixhon M, Adam C, et al. High prevalence of pituitary adenomas: a cross-sectional study in the Province of Liège, Belgium. J Clin Endocrinol Metab 2006;91:4769-75.

4. Heaney A. Management of aggressive pituitary adenomas and pituitary carcinomas. J Neurooncol 2014;117:459-68.

5. Kasuki L, Raverot G. Definition and diagnosis of aggressive pituitary tumors. Rev Endocr Metab Disord 2020;21:203-8.

6. Raverot G, Burman P, McCormack A, et al. European society of endocrinology clinical practice guidelines for the management of aggressive pituitary tumours and carcinomas. Eur J Endocrinol 2018;178:G1-G24.

7. Dai C, Feng M, Liu X, et al. Refractory pituitary adenoma: a novel classification for pituitary tumors. Oncotarget 2016;7:83657-68.

8. Dai C, Liu X, Ma W, et al. The treatment of refractory pituitary adenomas. Front Endocrinol (Lausanne) 2019;10:334.

9. Delellis RA. Pathology and genetics of tumours of endocrine organs. IARC Press, Lyon 2004.

10. Zada G, Woodmansee WW, Ramkissoon S, et al. Atypical pituitary adenomas: incidence, clinical characteristics, and implications. J Neurosurg 2011;114:336-44.

11. Chiloiro S, Doglietto F, Trapasso B, et al. Typical and atypical pituitary adenomas: a single-center analysis of outcome and prognosis. Neuroendocrinology 2015;101:143-50.

12. Zaidi HA, Cote DJ, Dunn IF, et al. Predictors of aggressive clinical phenotype among immunohistochemically confirmed atypical adenomas. J Clin Neurosci 2016;34:246-51.

13. WHO. Classifcation of tumours of endocrine organs. IARC Publications, Lyon 2017.

14. Laws ER, Penn DL, Repetti CS. Advances and controversies in the classification and grading of pituitary tumors. J Endocrinol Invest 2019;42:129-35.

15. Chatzellis E, Alexandraki KI, Androulakis II, et al. Aggressive pituitary tumors. Neuroendocrinology 2015;101:87-104.

16. Yokoyama S, Hirano H, Moroki K, et al. Are nonfunctioning pituitary adenomas extending into the cavernous sinus aggressive and/or invasive? Neurosurgery 2001;49:857-62; discussion 862-3.

17. Bao X, Deng K, Liu X, et al. Extended transsphenoidal approach for pituitary adenomas invading the cavernous sinus using multiple complementary techniques. Pituitary 2016;19:1-10.

18. de Aguiar PHP, Aires R, Laws ER, et al. Labeling index in pituitary adenomas evaluated by means of MIB-1: is there a prognostic role? A critical review. Neurol Res 2010;32:1060-71.

19. Raverot G, Vasiljevic A, Jouanneau E. Prognostic factors of regrowth in nonfunctioning pituitary tumors. Pituitary 2018;21:176-82.

20. Trouillas J, Roy P, Sturm N, et al. A new prognostic clinicopathological classification of pituitary adenomas: a multicentric case-control study of 410 patients with 8 years post-operative follow-up. Acta Neuropathol 2013;126:123-35.

21. Petry C, Poli JHZ, Dossin IA, et al. Evaluation of the potential of the Ki67 index to predict tumor evolution in patients with pituitary adenoma. Int J Clin Exp Pathol 2019;12:320-6.

22. Mete O, Asa SL. Clinicopathological correlations in pituitary adenomas. Brain Pathol 2012;22:443-53.

23. Ceccato F, Regazzo D, Barbot M, et al. Early recognition of aggressive pituitary adenomas: a single-centre experience. Acta Neurochir 2018;160:49-55.

24. Melmed S. Pathogenesis of pituitary tumors. Nat Rev Endocrinol 2011;7:257-66.

25. Reincke M, Sbiera S, Hayakawa A, et al. Mutations in the deubiquitinase gene USP8 cause Cushing's disease. Nat Genet 2015;47:31-8.

26. Melmed S. Pituitary-tumor endocrinopathies. N Engl J Med 2020;382:937-50.

27. Tatsi C, Stratakis CA. The genetics of pituitary adenomas. 
J Clin Med 2019;9:30.

28. Vasilev V, Daly AF, Trivellin G, et al. Hereditary endocrine tumours: current state-of-the-art and research opportunities: the roles of AIP and GPR101 in familial isolated pituitary adenomas (FIPA). Endocr Relat Cancer 2020;27:T77-T86.

29. Alband N, Korbonits M. Familial pituitary tumors. Handb Clin Neurol 2014;124:339-60.

30. Casanueva FF, Barkan AL, Buchfelder M, et al. Criteria for the definition of pituitary tumor centers of excellence (PTCOE): a pituitary society statement. Pituitary 2017;20:489-98.

31. Ciric I, Ragin A, Baumgartner C, et al. Complications of transsphenoidal surgery: results of a national survey, review of the literature, and personal experience. Neurosurgery 1997;40:225-36; discussion 236-7.

32. Kassam A, Snyderman CH, Mintz A, et al. Expanded endonasal approach: the rostrocaudal axis. Part I. Crista galli to the sella turcica. Neurosurg Focus 2005;19:E3.

33. Kassam A, Snyderman CH, Mintz A, et al. Expanded endonasal approach: the rostrocaudal axis. Part II. Posterior clinoids to the foramen magnum. Neurosurg Focus 2005;19:E4.

34. Kamiya-Matsuoka C, Cachia D, Waguespack SG, et al. Radiotherapy with concurrent temozolomide for the management of extraneural metastases in pituitary carcinoma. Pituitary 2016;19:415-21.

35. Chanson P, Dormoy A, Dekkers OM. Use of radiotherapy after pituitary surgery for non-functioning pituitary adenomas. Eur J Endocrinol 2019;181:D1-D13.

36. Lee CC, Sheehan JP. Advances in gamma knife radiosurgery for pituitary tumors. Curr Opin Endocrinol Diabetes Obes 2016;23:331-8.

37. Plitt AR, El Ahmadieh TY, Aoun SG, et al. Fractionated cyberknife stereotactic radiotherapy for perioptic pituitary adenomas. World Neurosurg 2019;126:e1359-e1364.

38. Puataweepong $\mathrm{P}$, Dhanachai M, Hansasuta A, et al. Outcomes for pituitary adenoma patients treated with linac-based stereotactic radiosurgery and radiotherapy: a long term experience in Thailand. Asian Pac J Cancer Prev 2015;16:5279-84.

39. Minniti G, Clarke E, Scaringi C, et al. Stereotactic radiotherapy and radiosurgery for non-functioning and secreting pituitary adenomas. Rep Pract Oncol Radiother 2016;21:370-8.

40. Ding D, Starke RM, Sheehan JP. Treatment paradigms for pituitary adenomas: defining the roles of radiosurgery and radiation therapy. J Neurooncol 2014;117:445-57.
41. Starke RM, Williams BJ, Jane JA, et al. Gamma Knife surgery for patients with nonfunctioning pituitary macroadenomas: predictors of tumor control, neurological deficits, and hypopituitarism. J Neurosurg 2012;117:129-35.

42. Darzy KH. Radiation-induced hypopituitarism. Curr Opin Endocrinol Diabetes Obes 2013;20:342-53.

43. Castinetti F. Radiation techniques in aggressive pituitary tumours and carcinomas. Rev Endocr Metab Disord 2020;21:287-92.

44. Ekeblad S, Sundin A, Janson ET, et al. Temozolomide as monotherapy is effective in treatment of advanced malignant neuroendocrine tumors. Clin Cancer Res 2007;13:2986-91.

45. Fadul CE, Kominsky AL, Meyer LP, et al. Long-term response of pituitary carcinoma to temozolomide. J Neurosurg 2006;105:621-6.

46. Lim S, Shahinian H, Maya MM, et al. Temozolomide: a novel treatment for pituitary carcinoma. Lancet Oncol 2006;7:518-20.

47. Burman P, Lamb L, McCormack A. Temozolomide therapy for aggressive pituitary tumours - current understanding and future perspectives. Rev Endocr Metab Disord 2020;21:263-76.

48. Trouillas J, Burman P, McCormack A, et al. Aggressive pituitary tumours and carcinomas: two sides of the same coin? Eur J Endocrinol 2018;178:C7-C9.

49. Knizhnik AV, Roos WP, Nikolova T, et al. Survival and death strategies in glioma cells: autophagy, senescence and apoptosis triggered by a single type of temozolomideinduced DNA damage. PLoS One 2013;8:e55665.

50. Zhang J, Stevens MF, Bradshaw TD. Temozolomide: mechanisms of action, repair and resistance. Curr Mol Pharmacol 2012;5:102-14.

51. Hegi ME, Diserens AC, Gorlia T, et al. MGMT gene silencing and benefit from temozolomide in Glioblastoma. N Engl J Med 2005;352:997-1003.

52. Stojic L, Brun R, Jiricny J. Mismatch repair and DNA damage signalling. DNA Repair 2004;3:1091-1101.

53. Bengtsson D, Schrøder HD, Andersen M, et al. Longterm outcome and MGMT as a predictive marker in 24 patients with atypical pituitary adenomas and pituitary carcinomas given treatment with temozolomide. J Clin Endocrinol Metab 2015;100:1689-98.

54. Kaltsas GA, Mukherjee JJ, Plowman PN, et al. The role of cytotoxic chemotherapy in the management of aggressive and malignant pituitary tumors. J Clin Endocrinol Metab 1998;83:4233-8. 
55. Donovan LE, Arnal AV, Wang SH, et al. Widely metastatic atypical pituitary adenoma with mTOR pathway STK11(F298L) mutation treated with everolimus therapy. CNS Oncol 2016;5:203-9.

56. Fukuoka H, Cooper O, Ben-Shlomo A, et al. EGFR as a therapeutic target for human, canine, and mouse ACTH-secreting pituitary adenomas. J Clin Investig 2011;121:4712-21.

57. Cooper O, Mamelak A, Bannykh S, et al. Prolactinoma

Cite this article as: Liu X, Dai C, Feng M, Li M, Chen G, Wang R. Diagnosis and treatment of refractory pituitary adenomas: a narrative review. Gland Surg 2021;10(4):1499-1507. doi: $10.21037 /$ gs-20-873
ErbB receptor expression and targeted therapy for aggressive tumors. Endocrine 2014;46:318-27.

58. Ortiz LD, Syro LV, Scheithauer BW, et al. Anti-VEGF therapy in pituitary carcinoma. Pituitary 2012;15:445-9.

59. Lin AL, Jonsson P, Tabar V, et al. Marked response of a hypermutated ACTH-secreting pituitary carcinoma to ipilimumab and nivolumab. J Clin Endocrinol Metab 2018;103:3925-30. 